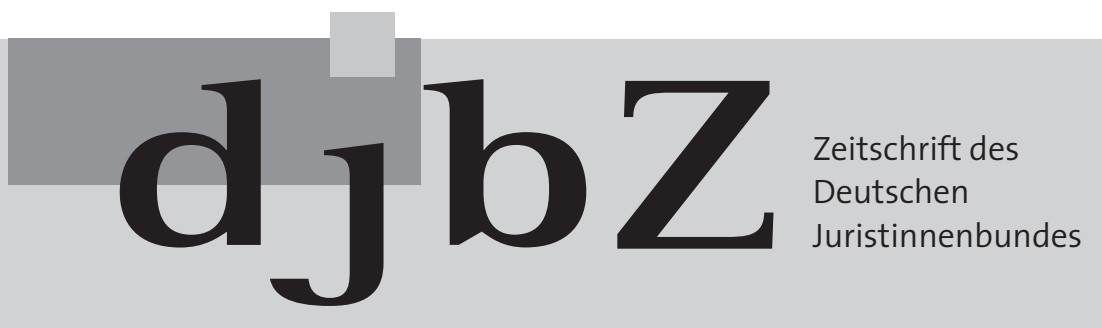

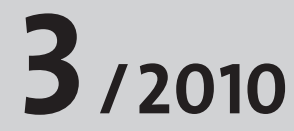

13. Jahrgang, Seite $97-146$

Herausgeber: Deutscher Juristinnenbund e. V.

Präsidium: Jutta Wagner, Rechtsanwältin und Notarin, Fachanwältin für Familienrecht, Berlin (Präsidentin); Renate Maltry, Rechtsanwältin, Fachanwältin für Familienrecht und Erbrecht, München; Ramona Pisal, Vorsitzende Richterin am Oberlandesgericht, Brandenburg a.d.H. (Vizepräsidentinnen); Dagmar Brinkmann, Regierungsdirektorin, Frankfurt a.M. (Schatzmeisterin); Schriftleitung: Anke Gimbal, Rechtsassessorin, Geschäftsführerin Deutscher Juristinnenbund, Berlin.

\title{
Liest du noch oder wirst du schon gelesen?
}

\section{Ein Bericht über die Arbeit als Chefredakteurin eines studentischen Online-Journals (Freilaw)}

\section{Claudia Tröller \\ Referendarin, Universität Freiburg i. Br.}

„Wer nur studiert, bekommt ein Problem“. So titelte der Spiegel in seiner Online-Ausgabe Ende letzten Jahres und berichtete zum ersten Mal über studentische Journale. Fachliche Kompetenz ist somit allein nicht mehr ausreichend. Bei der Vielzahl juristischer Absolvent(inn)en muss man sich aus der Masse durch Zusatzleistungen abheben. Was gewünscht wird, sind Qualifikationen und Erfahrungen wie beispielsweise die Arbeit in einem Verein, das Auslandssemester oder seltenere Sprachkenntnisse. Oder die Mitarbeit bei einem studentischen Journal.

Ich wurde auf Freilaw (zusammengesetzt aus „Frei“ für Freiburg und „law“ für Lawjournal) durch einen Kommilitonen meines Schwerpunktes aufmerksam, der mir die Idee beschrieb und mich einlud, bei einer Sitzung dabei zu sein. Positiv überrascht hat mich die Begeisterung der Redaktionsmitglieder, die sich neben ihrem Studium für eine so zeitintensive Sache wie dem Aufbau einer Zeitschrift engagierten.

Zunächst war ich hauptsächlich im Ressort Technik tätig, da neben der redaktionellen Mitarbeit jedes Mitglied der Redaktion einen weiteren Aufgabenbereich (Werbung, Layout, Human Resources, Technik) übernimmt. Ich fand es interessant, eine Website aufzubauen, zu betreuen und auch zu verändern. Dass dies sehr viel Zeit in Anspruch nimmt, habe ich spätestens an langen Wochenenden vor Veröffentlichung der Zeitschrift gemerkt, wenn die Artikel auf die Seite gesetzt werden mussten.

Als ich zur Redaktion stieß, befand sich die Zeitschrift gerade im Aufbau. Es waren zahlreiche Gespräche mit Professor(inn)en und dem Dekan nötig, um die Zeitschrift als offizielle Fakultätszeitschrift anerkennen zu lassen und Unter- stützung seitens der Professor(inn)en zu erhalten, da das Projekt etwas ganz Neues war. Mit den fortschreitenden Ausgaben etablierte sich die Zeitschrift und es wurde bei Werbeaktionen immer weniger die Frage gestellt, was Freilaw eigentlich sei.

Ein Höhepunkt war die Gründung des tragenden Vereins. Davor war es nur ein loser Zusammenschluss von Student(inn) en, nun erhielt unser studentisches Journal eine offizielle Form. Die damit verbunden Wege der Bürokratie und die verschiedenen Genehmigungen waren zum Teil ein nervenaufreibendes Unternehmen. Das alles konnte nach Erhalt der endgültigen Bescheinigung jedoch nur als Erfolg gewertet werden.

Als die damalige Chefredakteurin aufgrund ihrer Examensvorbereitung ausscheiden musste, wechselte ich in die Chefredaktion. Schnell kam allerdings die Erkenntnis, dass eine Zeitschrift zu leiten nicht nur bedeutet, Artikel zu veröffentlichen, sondern sich im großen Maße auch um die Artikel zu kümmern und Student(inn)en zu motivieren. Ein Ausgabenschwerpunkt kann viele Student(inn)en ansprechen und zu vielen Artikeln anregen, für einen anderen waren schon einmal Kontakte in die Schweiz nötig, um eine vollständige Ausgabe zu veröffentlichen. Zudem kommen noch weitere organisatorische Aufgaben wie die Werbung neuer Redaktionsmitglieder (neben nur schreibenden Autor(inn)en), die Koordination der einzelnen Ressorts sowie aktuelle Werbeaktionen hinzu.

Zur Arbeit gehört auch die Vorbereitung der im zweiwöchigen Rhythmus stattfindenden Redaktionssitzungen. Sie dienen hauptsächlich dazu, neue Projekte und Ideen zu entwickeln und die Artikel für die kommende Ausgabe zu besprechen. Ansonsten braucht es für eine Sitzung nicht viel: einen Raum, ein Team, eine Tagesordnung und Ideen. In diesem Rahmen werden auch Neuerungen beschlossen wie beispielsweise ein monatlicher Stammtisch für Redaktionsmitglieder 
und interessierte Student(inn)en oder das Bedrucken von Werbekugelschreibern, um die Zeitschrift unter Student(inn)en bekannter zu machen.

Etwas Neues war für mich auch die Verantwortung, die man mit der Veröffentlichung der Zeitschrift für die darin enthaltenen Texte übernimmt. Am Anfang war es nicht leicht, Texte in der ursprünglichen Form ohne redaktionelle Änderungen zu veröffentlichen oder sehr vom allgemeinen Verständnis abweichende Meinungen zuzulassen. Mit jeder weiteren Ausgabe war dies aber besser einzuschätzen, sodass auch heute auch Texte unbequemer Autor(inn)en ihren Platz finden.

Manche Stilblüten und Berichte von Autor(inn)en konnten trotz allem nicht veröffentlicht werden. Ein Artikel aus Amerika beispielsweise, der ein Loblied auf den zweiten Verfassungszusatz (das Recht der Amerikaner, Waffen zu tragen) sang, ohne eine andere Meinung zuzulassen oder eine wissenschaftliche Auseinandersetzung damit anzuregen. Oder ein finnischer Beitrag, der in einem solch schlechten Englisch geschrieben war, dass der zuständige Lektor nach der ersten Seite das Handtuch geworfen hat. Gerade nach diesen Erlebnissen ist man positiv überrascht, wenn ein(e) Student(in) sich mit einem Thema (außerhalb seines (ihres) Schwerpunktes, nur aus Interesse) beschäftigt hat und zwanzig Seiten fundiertes Wissen einreicht mit der Bitte um Veröffentlichung.

Während meiner eigenen Examensvorbereitung habe ich weiterhin aktiv mitgewirkt. Für mich war es ein Ausgleich zum theoretischen Wissen und ich konnte mich auch weiterhin einbringen und die Diskussion suchen. Erfreulich ist, dass dabei auf gleicher Ebene bei studentischen Gruppen diskutiert wird, über Semester- und Geschlechtergrenzen hinweg. Anfänger(innen) werden im gleichen Maße respektiert wie die Gründungsmitglieder. Student(inn)en sind in dieser Hinsicht sehr tolerant und unvoreingenommen. Und so ist es möglich, verschiedene Vorschläge einzubringen und gemeinsam weiter zu entwickeln.

Die Chefredaktion gab mir die Möglichkeit, die Ausgaben und das Konzept des Journals mitzugestalten und auch meine Vorstellungen maßgeblich einzubringen. Man lernt dabei auch, seine Meinung zu vertreten und sich für das einzusetzen, was einem wichtig ist. Ich kann nur jeder Studentin raten, sich eine Aufgabe neben dem Studium zu suchen, für die sie sich interessiert und sich persönlich einsetzen will. Sich schon als Studentin zu engagieren, ist ein großer Gewinn.

\section{Wie gründe ich einen feministischen Lesekreis? - Eine Anleitung zum Selbermachen}

\author{
cand. iur. Sophie Arndt \\ Studentin, Freiburg i. Br.
}

\section{Dr. Anna Katharina Mangold, LL.M. (Cambridge) \\ Wissenschaftliche Mitarbeiterin, Freiburg i. Br.}

Man nehme eine Handvoll interessierte Studierende, vorzugsweise beiderlei Geschlechts, sammle an abgelegenen Orten im Rahmen des Jurastudiums üblicherweise vernachlässigte Autoren bzw. - ganz überwiegend - Autorinnen, okkupiere einen für gelegentlich hitzige Diskussionen geeigneten Raum und schon kann es losgehen. In Freiburg, einer inzwischen auch sonst für innovative feministische Projekte bekannten Universität (Justitia Mentoring), haben wir genau das gemacht - und kurzerhand einen feministischen Lesekreis gegründet.

Bei einem gemeinsamen Mittagessen von Teilnehmerinnen des Frauenförderungsprogrammes Justitia Mentoring fanden sich im Sommersemester 2009 eine Studentin und eine Doktorandin zusammen und beschlossen, fürderhin feministische Texte gemeinsam zu lesen und zu diskutieren. Über persönliche Ansprache und Rundmails konnten weitere Mitstreiterinnen geworben werden. Flugs eine Mailing-Liste angelegt, und schon wurde munter über das Thema der ersten Sitzung debattiert.
„Feministische Rechtswissenschaft" von Lena Foljanty und Ulrike Lembke (Nomos 2006) bot dank ausführlicher Literaturhinweise einen hervorragenden Einstieg. Die erste Sitzung war der Lektüre von grundlegenden Texten gewidmet, die der Frage nachgingen, was denn feministische Rechtswissenschaft überhaupt sei. Doch schon in den nächsten Sitzungen begannen wir, gewappnet mit einschlägiger Hintergrundlektüre, neuere Entscheidungen des Bundesverfassungsgerichts feministisch zu analysieren. Dem Transsexuellen-Urteil (BVerfGE 121, 175) gewannen wir im Kontrast mit Michel Foucaults "Sexualität und Wahrheit“ und Judith Butlers „Gender Trouble“ ganz neue Dimensionen ab. Das InzestUrteil (BVerfGE 120, 224) kontrastierten wir mit Auszügen aus Lévi-Strauss, „Die elementaren Strukturen der Verwandtschaft" und weiteren Untersuchungen von Judith Butler zum Inzest-Tabu.

In den folgenden Sitzung ging es dann etwa um solche Themen: Welche Rolle spielt Geschlecht im Strafrecht? Hier gab es sowohl Ansätze und Arbeiten aus der kritischen Kriminologie zu studieren, wie auch solche, die sich kritisch mit den scheinbar (geschlechts-)neutralen Begrifflichkeiten des Strafrechts auseinandersetzen und danach fragen, inwieweit derartige Vorannahmen das Strafrecht prägen und - umgekehrt welche Auswirkungen das Verständnis von Kriminalität seinerseits auf Geschlechterrollen hat. „Typischer“ feministisch 